\title{
Klebsiella pneumoniae co-producing KPC and RmtG, finally targeting Switzerland
}

\author{
Stefano Mancini a,b,c,*, Laurent Poirel a,b,c , Myriam Corthesy ${ }^{\mathrm{d}}$, Gilbert Greub ${ }^{\mathrm{d}}$, Patrice Nordmann ${ }^{\mathrm{a}, \mathrm{b}, \mathrm{c}, \mathrm{d}}$ \\ a Emerging Antibiotic Resistance Unit, Medical and Molecular Microbiology, Department of Medicine, University of Fribourg, Fribourg, Switzerland \\ ${ }^{\mathrm{b}}$ French INSERM European Unit, University of Fribourg (LEA-IAME), Fribourg, Switzerland \\ c Swiss National Reference Center for Emerging Antibiotic Resistance, Fribourg, Switzerland. \\ ${ }^{\mathrm{d}}$ Institute for Microbiology, University of Lausanne and University Hospital Center, Lausanne, Switzerland
}

\begin{abstract}
A carbapenem- and pan-aminoglycoside-resistant Klebsiella pneumoniae strain was isolated from a Brazilian patient hospitalized in a Swiss hospital. The clinical isolate carried genes encoding the KPC-2 carbapenemase and the RmtG 16S rRNA methyltransferase. This is the first report of a carbapenem-resistant K. pneumoniae producing RmtG in Europe.
\end{abstract}

Klebsiella pneumoniae is a leading cause of hospital-associated bacterial infections. The acquisition of a multidrug resistance profile has been associated to the predominance of some specific sequence types (STs) in clinical settings. Among the main threats, strains producing carbapenemases are considered as the most worrying ones. In this regard, carbapenem-resistant K. pneumoniae strains of clonal complex 258 (CC258 that includes ST11, ST258, ST340 and ST437) that produce KPC-type carbapenemases have disseminated worldwide and have been often responsible for nosocomial outbreaks (Woodford et al., 2011). Worryingly, the co-occurrence of $16 S$ rRNA methyltransferases, such as ArmA, RmtB, RmtD, and RmtG enzymes, that confer high-level and broad-spectrum resistance towards aminoglycosides has been increasingly reported among KPC-producing $K$. pneumoniae in recent years (Cerdeira et al., 2016; Cheng et al., 2016; Quiles et al., 2015). Once KPC and a 16S rRNA methyltransferase are co-produced, it almost leads to pandrug resistance. We report here the emergence of a K. pneumoniae of clonal complex 258 co-producing KPC-2 and RmtG in Switzerland.

K. pneumoniae strain KP05-2017 was isolated from a rectal swab upon admission in May 2017 in a Swiss hospital. It was recovered from a 73-year-old patient who had been hospitalized in Brazil the month before for treatment of a hemorrhagic shock caused by perforation of a gastric ulcer.

Disk diffusion assay (Sanofi-diagnostic Pasteur, France) was performed to test the antimicrobial susceptibility while E-test (bioMérieux, La Balme-les-Grottes, France), and broth dilution techniques were used to assess minimal inhibitory concentrations (MIC). Antimicrobial susceptibility was interpreted according to the CLSI guidelines. Isolate

\footnotetext{
* Corresponding author. Tel.: +41-26-300-9583.

E-mail address: stefano.mancini@unifr.ch (S. Mancini).
}

KP05-2017 was resistant to ceftriaxone (MIC of $6 \mathrm{mg} / \mathrm{L}$ ), but remained susceptible to ceftazidime and cefepime (MIC of 3 and $8 \mathrm{mg} / \mathrm{L}$, respectively). It exhibited resistance to imipenem, ertapenem and meropenem with MICs $<32 \mathrm{mg} / \mathrm{L}$. Carbapenemase activity was assessed using the Carba NP test (Nordmann et al., 2012). Since the isolate was resistant to amikacin, gentamicin, tobramycin and netilmicin, the occurrence of a 16S rRNA methyltransferase was tested and confirmed by using the Rapid Aminoglycoside NP test that is based on a rapid culture of bacteria in the presence of defined concentrations of gentamicin and amikacin (Nordmann et al., 2017). Isolate KP05-2017 was also resistant to sulfonamide, nalidixic acid, ciprofloxacin, chloramphenicol, tetracycline, trimethoprim-sulphamethoxazole and the combination of ceftolozane-tazobactam (MIC $24 \mathrm{mg} / \mathrm{L}$ ). It remained susceptible only to fosfomycin and exhibited MIC values of colistin, the ceftazidimeavibactam association and tigecycline at $2 \mathrm{mg} / \mathrm{L}, 1.5 \mathrm{mg} / \mathrm{L}$, and $0.38 \mathrm{mg} / \mathrm{L}$, respectively.

Multiplex PCRs performed to detect Ambler class A, B and D genes and 16S rRNA methyltransferase genes (Bercot et al., 2011; Poirel et al., 2011) followed by sequencing revealed the presence of the $b l a_{\mathrm{KPC}-2}$ and $r m t G$ genes. Analysis of the plasmid content obtained with the Kieser method (Kieser, 1984) identified three plasmids of ca. 10, 45 and $150 \mathrm{~kb}$ in size. Molecular profiling showed that KP05-2017 belongs to the sequence type 11 . Mating-out assays were performed to establish the transferability of the $b a_{\mathrm{KPC}-2}$ and $r m t G$ genes using the azide-resistant Escherichia coli J53 as recipient strain. Transconjugants were selected on LB agar plates containing sodium azide $(100 \mathrm{mg} / \mathrm{L})$, amikacin $(50 \mathrm{mg} / \mathrm{L})$ and gentamicin $(50 \mathrm{mg} / \mathrm{L})$, indicating the transferability of the ca. 45-kb plasmid carrying the $r m t G$ gene. However, no transconjugant was obtained when selecting on imipenem $(1 \mathrm{mg} / \mathrm{L})$.

Genomic DNA was subjected to whole genome sequencing using an Illumina MiniSeq platform (Illumine, San Diego, CA, USA), which 
generated 4.564 .240 reads with an average size of $144.3 \mathrm{bp}$. De novo assembling of the reads was performed using CLC Genomics Workbench version 7.5.1 (Qiagen, France). The final size of the draft genome was of $5.779 .331 \mathrm{bp}$, with an average CG content of $56.8 \%$.

The analysis of the resistome using ResFinder (https://cge.cbs.dtu. $\mathrm{dk} /$ services/ResFinder/) identified genes conferring resistance to $\beta$ lactams ( $b l a_{\mathrm{TEM}-1 \mathrm{C}}$ and $b l a_{\mathrm{KPC}-2}$ ), aminoglycosides (aadA2, aadA5, strA and $r m t G$ ), macrolides, lincosamides and streptogramin B [erm(B) and $m p h(A)$ ], phenicols (cmlA1), sulphonamides (sul1 and sul2), trimethoprim (dfrA14, dfrA15 and drfA17) and tetracycline [tet(D)]. Plasmid finder (https://cge.cbs.dtu.dk/services/PlasmidFinder/) revealed the presence of the IncFIB(Mar) and IncB/O replicons, with the latter one located in the $48 \mathrm{kB}$ plasmid carrying the $r m t G$ gene. PHASTER (http:// phaster.ca/) unveiled the presence of four intact, four incomplete and three putative prophages.

The successful and worldwide dissemination of KPC-2 producing K. pneumoniae strains belonging to CC258, which includes sequence type 11 , is now well established. The emergence of clones coproducing 16S rRNA methyltransferases further complicates the treatment of infections due to these pathogens, with extremely limited therapeutic options left. The occurrence in Switzerland of a K. pneumoniae clone co-producing KPC-2 and RmtG, with the latter enzyme being so far reported in America and India (Bueno et al., 2013; Filgona et al., 2015; Hu et al., 2014; Poirel et al., 2014), underlines how importation of multidrug-resistant clones may occur despite the high standards of antibiotic stewardship programs and hygiene control in effect in the Swiss healthcare institutions.

Of note, and despite elevated MICs, isolate KP05-2017 remained susceptible to ceftazidime and cefepime, suggesting a low-level of expression of the $b l a_{\mathrm{KPC}-2}$ gene. Notwithstanding, isolate KP05-2017 could grow on CHROMagar mSuperCarba chromID ${ }^{\circledR}$ (CHROMagar, - Paris, France), CARBA SMART and CHROMID ${ }^{\circ}$ ESBL media (bioMérieux, La Balme-les-Grottes, France) (data not shown), indicating that such isolate, despite exhibiting low MICs of some broad-spectrum cephalosporins, may be well detected with those screening media when used for screening of carriers.

\section{Funding}

This work was funded by the University of Fribourg and by the Swiss National Reference Center for Emerging Antibiotic Resistance (NARA).
Accession number. The draft genome sequence of the K. pneumoniae KP05-2017 has been deposited to the GenBank under the accession number NSLD00000000.

\section{Transparency Declarations}

None to declare.

\section{References}

Bercot B, Poirel L, Nordmann P. Updated multiplex polymerase chain reaction for detec tion of 16S rRNA methylases: high prevalence among NDM-1 producers. Diagn Microbiol Infect Dis 2011;71(4):442-5

Bueno MF, Francisco GR, O'Hara JA, de Oliveira Garcia D, Doi Y. Coproduction of 16S rRNA methyltransferase RmtD or RmtG with KPC-2 and CTX-M group extended-spectrum beta-lactamases in Klebsiella pneumoniae. Antimicrob Agents Chemother 2013;57(5): 2397-400.

Cerdeira L, Fernandes MR, Francisco GR, Bueno MF, Ienne S, Souza TA, et al. Draft genome sequence of a hospital-associated clone of Klebsiella pneumoniae ST340/CC258 coproducing RmtG and KPC-2 isolated from a pediatric patient. Genome Announc 2016;4(6): $01130-16$.

Cheng L, Cao XL, Zhang ZF, Ning MZ, Xu XJ, Zhou W, et al. Clonal dissemination of KPC-2 producing Klebsiella pneumoniae ST11 clone with high prevalence of oqxAB and rmtB in a tertiary hospital in China: results from a 3-year period. Ann Clin Microbiol Antimicrob 2016:15:1.

Filgona J, Banerjee T, Anupurba S. Incidence of the novel $r m t F$ and $r m t G$ methyltransferases in carbapenem-resistant Enterobacteriaceae from a hospital in India. J Infect Dev Ctries 2015;9(9):1036-9.

Hu F, Munoz-Price LS, DePascale D, Rivera JI, Doi Y. Klebsiella pneumoniae sequence type 11 isolate producing RmtG 16S rRNA methyltransferase from a patient in Miami, Florida. Antimicrob Agents Chemother 2014;58(8):4980-1.

Kieser T. Factors affecting the isolation of CCC DNA from Streptomyces lividans and Escherichia coli. Plasmid 1984;12(1):19-36.

Nordmann P, Jayol A, Dobias J, Poirel L. Rapid aminoglycoside NP test for rapid detection of multiple aminoglycoside resistance in Enterobacteriaceae. J Clin Microbiol 2017; 55(4):1074-9.

Nordmann P, Poirel L, Dortet L. Rapid detection of carbapenemase-producing Enterobacteriaceae. Emerg Infect Dis 2012;18(9):1503-7.

Poirel L, Labarca J, Bello H, Rioseco ML, Bernabeu S, Nordmann P. Emergence of the 16S rRNA methylase RmtG in an extended-spectrum-beta-lactamase-producing and colistin-resistant Klebsiella pneumoniae isolate in Chile. Antimicrob Agents Chemother 2014;58(1):618-9.

Poirel L, Walsh TR, Cuvillier V, Nordmann P. Multiplex PCR for detection of acquired carbapenemase genes. Diagn Microbiol Infect Dis 2011;70(1):119-23.

Quiles MG, Rocchetti TT, Fehlberg LC, Kusano EJ, Chebabo A, Pereira RM, et al. Unusual association of NDM-1 with KPC-2 and armA among Brazilian Enterobacteriaceae isolates. Brazilian journal of medical and biological research. Rev Bras Pesqui Med Biol 2015;48(2):174-7.

Woodford N, Turton JF, Livermore DM. Multiresistant gram-negative bacteria: the role of high-risk clones in the dissemination of antibiotic resistance. FEMS Microbiol Rev 2011;35(5):736-55. 